\title{
Pain Scale: When the Training Influences Its Use
}

\author{
Zaira Moura da Paixão Freitas ${ }^{1}$, Carlos Umberto Pereira², Débora Moura da Paixão Oliveira ${ }^{1}$ \\ ${ }^{1}$ Health Science, University Federal of Sergipe, Sergipe, Brazil \\ ${ }^{2}$ Department of Medicine, University Federal of Sergipe, Sergipe, Brazil \\ Email: zairampfreitas@yahoo.com.br
}

How to cite this paper: da Paixão Freitas, Z.M., Pereira, C.U. and da Paixão Oliveira, D.M. (2018) Pain Scale: When the Training Influences Its Use. Open Journal of Nursing, 8, 130-138.

https://doi.org/10.4236/ojn.2018.82011

Received: December 20, 2017

Accepted: February 10, 2018

Published: February 13, 2018

Copyright (C) 2018 by authors and Scientific Research Publishing Inc. This work is licensed under the Creative Commons Attribution International License (CC BY 4.0).

http://creativecommons.org/licenses/by/4.0/

\begin{abstract}
The aim of this study was to determine the level of knowledge that nurses in the neonatal intensive care units (NICU) of a public birth center had about the use of the Neonatal Infant Pain Scale (NIPS) and to test how their scoring for NIPS changed before and after training. Thirty nurses applied the NIPS scale to newborns that were procedures considered painful. During the first and second evaluations, nurses diagnosed 30\% infants as having pain and 70\% infants as having an absence of pain. In the third and fourth evaluations, after the NIPS parameters had been explained, we observed an increase in the number of infants diagnosed with the presence of pain (65\%). The results indicate the importance of formal training for the systemic evaluation of pain in newborns.
\end{abstract}

\section{Keywords}

Pain, Pain Scale, Nurse

\section{Introduction}

For many years, it was believed that newly born infants particularly pre-term infants, felt no pain due to their neurological immaturity and inability to express emotions. Nevertheless, research has shown that neonates can and do experience pain [1].

During their hospitalization in neonatal intensive care units (NICU), at-risk newborns are exposed to noise, continuous and intense light, and potentially painful invasive clinical procedures [1] [2]. However, approximately $50 \%$ of pre-term newborns do not cry in response to a painful stimulus. These infants' responses may differ from those of full-term infants. These infants may instead present physiological responses such as increased cardiac and respiratory fre- 
quencies, increased intracranial and arterial pressure, variable oxygen saturation of the blood, increased hormone levels and decreased insulin levels in response to painful stimuli [3].

The sick newly born or pre-term infant presents an imbalance between subsystems autonomic, motor, behavioral states, attention-interaction and regulator, when subjected to painful procedures or an overstimulation. This imbalance in the autonomic system may cause the change in breathing patterns and bowel movements. Disorganized movements and changes in muscle tone can occur by instability in the motor system. In the system of behavioral state, instability results in changes in sleep-wake cycle resulting lack of sleep and energy loss [4].

As physiological responses to painful stimulus, there are changes in neuroendocrine, respiratory and cardiovascular systems. In the neuroendocrine system evidenced hormonal changes are involving the pituitary, adrenal and pancreas, causing disturbances in the metabolism of proteins and carbohydrates [5]. As biochemical changes, variations in metabolism such as hyperglycemia, increased release of cortisol, catecholamines, glucagon, interleukin, growth hormone, endorphins, renin, angiotensin II, aldosterone, antidiuretic hormone and decreased secretion of insulin and testosterone. Bioelectric variations also occur as changes in the electroencephalogram and electrocardiogram [1] [5].

In the cardiovascular system, there is observed increase in cardiac output as a result of increased heart rate, increased myocardial oxygen consumption, arrhythmias, hypertension, tachycardia, and changes in intracranial pressure and decreased oxygen saturation [1]. In respiratory system, the increase was observed in respiratory rate, oxygen consumption, mean airway pressure in respiratory tract and muscle tone [4].

The pain is also associated with changes in behavior with changes in facial expression, body movements, crying and changes in autonomic symptoms such as tachypnea, hyperhidrosis, mydriasis, flushing, pallor and muscle tension [1] [4]. The evaluation and measurement of pain are important components of the clinical management of pain. The appropriate evaluation of pain in diagnosis allows clinicians to determine whether intervention is needed, whether a treatment has been effective and to identify the care that is most effective in alleviating the patient's symptom [1]. These observations illustrate why health care professionals must be able to recognize neonatal pain.

Published literature mentions several scales that can be used to measure pain in infants, one of which is the Neonatal Infant Pain Scale (NIPS). This scale comprises six pain indicators, five behavioral and one physiological. Specifically, the parameters evaluated are facial expression, crying, respiratory standard, movements of arms, movements of legs and consciousness. It is useful in evaluating pain in both full-term and premature newborns, making it possible to differentiate between painful and non-painful stimuli in these children [6]. The evaluations are made in one minute intervals before, during and after the aggressive procedure [7]. It is not recommended their use in isolation and should 
be taken into account the general state of the newborn and the environment where it is inserted. The total score can range from 0 to 7 . For each score we have a specific meaning: the score 0 means the absence of pain, a score 1 to 2 means the presence of weak pain, the score 3 to 5 means the presence of moderate pain and scores between 6 and 7 means the presence of severe pain [7].

Over the past years, much work has been carried out to develop tools to evaluate pain in the NICU and many scales, based on different pain indicators, have been developed for this purpose. These scales should be used according to different circumstances.

The Premature Infant Pain Profile (PIPP), developed by Stevens et al., is composed of seven behavioral, physiological, and contextual indicators, has established validity and reliability. It was developed to assess acute pain in preterm and term neonates [8]. The indicators are gestational age, behavioral state, heart rate, oxygen saturation, brow bulge, eye squeeze, and nose labial furrow, each measured from 0 to 3 , with the total score ranging from 0 to 21 . A total score of 6 or under indicates no pain; 12 or more points indicate moderate to severe pain [7] [8].

The CRIES (Crying, Requires $\mathrm{O}_{2}$ for saturation above 95\%, increased vital signs-heart rate and blood pressure, Expression, Sleepless) was developed by Krechel and Bildner to measure postoperative pain in preterm and term infants, has established validity and inter-rater reliability. Each of the five items is scored from 0 to 2, the total score ranging from 0 to 10 [7] [9].

The Neonatal Facial Coding System (NFCS) was developed by Grunau and Craig on 1987. The indicators are brow bulge, eyes squeezed, nose labial furrowing and mouth open to relate the pain stimulus. This coding system is sensitive to acute pain in premature, term-born neonates and infants up to 18 months of age [7] [9].

The scale Pain Assessment in Neonates (PAIN) assesses acute pain in preterm and term infants during routine painful procedures, through indicators like facial expression, cry, breathing pattern, movement of the extremities, alert state, supplemental oxygen required and heart rate increased [10].

The CONFORT scale was developed to measure postoperative pain in intensive care environment to assess the distress and comfort in ventilated children. This scale comprises eight indicators. Six of the indicators are behavioral ones (alertness, calmness, muscle tone, movement, facial tension, and respiratory response/crying), and two are physiological indicators: heart rate and mean arterial pressure [9].

The evaluation of neonatal pain is a difficult task for health professionals, but with the appropriate technical and scientific knowledge and a precise evaluation tool, pain evaluation will be feasible. Empiricism and under-treatment can be avoided, and pain level can become a fifth vital criterion used to promote neonatal welfare.

This study aimed was to determine the level of knowledge that nurses in the 
NICU of a public birth center had about the use of the NIPS pain scale and to test how their scoring for NIPS changed before and after training.

\section{Methods}

The NICU of a public birth center in Aracaju County, has 10 beds, with terciary level of acuity, pre-term newborns is the population served. The NIPS scale was chosen compared to other tools, particularly for the NICU, because it was already an usual way to assessed the pain in use at the NICU.

A quantitative survey was performed at the NICU. Thirty nurses in the unit took part in the research from December 2009 to May 2010. They have a choice to participate or no. All agreed to participation. The nurses were blinded to any aspect of the study purpose or methods.

A questionnaire was given to gather personal and professional data about the nurses and determine their ability to evaluate pain in newborns using the NIPS scale. All data were collected at the birth center.

The nurses applied the NIPS scale to newborn infants during procedures like intravenous catheter insertion, removal of adhesive tapes, heel stick, gastric suction, endotracheal suctioning, rectal tube insertion, endotracheal extubation, gavage, nebulization, diaper changes, physiotherapy maneuvers, dressing change or postural changes. This procedures were considered painful. Thirty infants were selected for participation randomly. It was excluded newborns with neurological diseases, clinical instability in the respiratory or cardiovascular system or those who received analgesics or anesthetic before the procedure.

Once the evaluation was complete, the nurse received explanations about the parameters used to interpret the pain. Completing this explanation, further evaluations were performed by nurses in the same children. The same infants were evaluated by the same nurses before and after training aiming to verify the influence of clarifying the interpretation of parameters in the recognition of pain during the procedure. Each nurse completes five evaluations.

A score of zero it was considered no pain, one to two, as weak pain, three to five as moderate pain and six to seven as severe pain.

Even if the nurse has knowledge about the situation in which there was the newly born infants, it is considered hit when the professional diagnosed the presence of pain during the procedure becomes invasive, aggressive, uncomfortable and potentially painful and occurring variability of oxygen saturation and increased heart rate in newly born infants or if the professional diagnosed the absence of pain when the child was subjected to non-painful procedures, with no variation in oxygen saturation and heart rate. The error was considered that, even during painful procedures, the professional diagnosed the absence of pain, or when during painless procedures, there was the diagnosis of the presence of pain.

To avoid bias of change in diagnosis, it was considered the proximity of the cradles and it was respected the one-minute interval between the explanation of 
scale parameters and reassessment of the child, based on NIPS scale.

Even if the nurse has knowledge about the situation in which there was the newly born infants, it was considered able to perceive the symptom when the professional identified the presence of pain during the procedure becomes invasive, aggressive, uncomfortable and potentially painful and occurring variability of oxygen saturation and increased heart rate in newly born infants or if the professional identified the absence of pain when the child was subjected to non-painful procedures, with no variation in oxygen saturation and heart rate. The disability was considered that, even during painful procedures, the professional identified the absence of pain, or when during painless procedures, there was the identification of the presence of pain.

The project was approved by the Ethics Committee of University Federal of Sergipe, identification number (0998.0.000.107 - 08, June 2008). The nurses and parents of newborns agreed to participate in the study and signed the Term of Free and Clear Consent, guaranteeing that the study was ethical, based on the Edict of Regulation 196/96 of the Council of National Health of the Federal Health Department.

Pearson Chi-square or Fisher's exact statistical tests were used to verify nurses' knowledge of the evaluation and treatment of pain in neonates (using SPSS 16.0). The McNemar statistical test was used to compare nurses' recognition of pain before and after explanations.

\section{Results}

\section{Characterization of the Nurses}

\section{Socio-Demographic Data}

The study group was composed of the 30 nurses of NICU. All subjects were female, with an average age of $32.9 \pm 6.9$ years (minimum 23, maximum 46). Twenty nurses $(66.6 \%)$ had taken graduate specialization in public health, five (16.6\%) had not and five (16.6\%) were currently taking it. It was clear that none of the professionals had any specialization in pediatrics, neonatology or master course. The education background and work experiences of the nurses ranged from seven to nine years.

They recognized the neonatal pain and used behavioral and physiological parameters to assess it, but they didn't know scales for pain assessment. The failure to use the NIPS scale was promoted by the lack of parameters for its interpretation during the recognition of pain. Additional socio-demographic data are shown in Table 1.

Table 2 shows that the majority of the nurses $(n=21,70 \%)$ were not aware of any scale that could be used to evaluate the neonatal pain, while just nine nurses (30\%) knew of at least one scale and a maximum of three scales. The NIPS, PIPP (Premature Infant Pain Profile), CRIES (Neonatal Postoperative Pain Evaluation Score), NFCS (Neonatal Facial Coding System) and PAIN (Pain Assessment in Neonates) scales, as well as the COMFORT scale of sedation (COMFORT Score), were familiar to some of the nurses. 
Table 1. Characterization of nurses in this study according to age, number of children, graduation time and length of employment in the chosen NICU.

\begin{tabular}{|c|c|c|c|}
\hline Variables & Nurses & $\begin{array}{c}\text { Absolute } \\
\text { Frequency }(\mathrm{N})\end{array}$ & $\begin{array}{c}\text { Percentage of } \\
\text { Sample (\%) }\end{array}$ \\
\hline \multirow[t]{3}{*}{ Age (years) } & 20 to 29 & 8 & 26.7 \\
\hline & 30 to 39 & 17 & 56.6 \\
\hline & 40 to 49 & 5 & 16.7 \\
\hline \multirow[t]{6}{*}{$\mathrm{N}^{\circ}$ of children } & 0 & 14 & 46.7 \\
\hline & 1 & 9 & 30.0 \\
\hline & 2 & 5 & 16.7 \\
\hline & 3 & 1 & 3.3 \\
\hline & More than 3 & 1 & 3.3 \\
\hline & Less than 1 & 1 & 3.3 \\
\hline \multirow{5}{*}{$\begin{array}{l}\text { Time since learned a } \\
\text { university degree (years) }\end{array}$} & 1 to 3 & 9 & 30.0 \\
\hline & 4 to 6 & 4 & 13.3 \\
\hline & 7 to 9 & 11 & 36.7 \\
\hline & 10 and more & 5 & 16.7 \\
\hline & Less than 1 & 8 & 26.6 \\
\hline \multirow{3}{*}{$\begin{array}{l}\text { Time of work in the } \\
\text { NICU (years) }\end{array}$} & 1 to 3 & 15 & 50.0 \\
\hline & 4 to 6 & 4 & 13.4 \\
\hline & 7 to 9 & 3 & 10.0 \\
\hline
\end{tabular}

Table 2. Nurses' knowledge of scales used to evaluate neonatal pain, Aracaju, 2010.

\begin{tabular}{ccc}
\hline Variables & $\begin{array}{c}\text { Absolute } \\
\text { Frequency }(\mathrm{n})\end{array}$ & $\begin{array}{c}\text { Relative } \\
\text { Frequency (\%) }\end{array}$ \\
\hline Do not know any scales & 21 & 70.0 \\
Know of 1 scale & 6 & 20.0 \\
Know of 2 scales & 2 & 6.7 \\
Know of 3 scales & 1 & 3.3 \\
\hline
\end{tabular}

In the first and second evaluations in which nurses used the NIPS scale without an explanation of the parameters used for its interpretation, 18 (30\%) infants were identified with pain, and $42(70 \%)$ were identified as having no pain.

In the third and fourth evaluations, after nurses had received an explanation of the parameters of the NIPS scale, we observed an increase in the number of children identified with pain $(\mathrm{n}=39,65 \%)$ and a decrease in the number of children identified with no pain $(n=21,35 \%)$.

There was a statistically significant difference in the nurses' responses between evaluations 1 and 2 and evaluations 3, 4 and 5, which indicates that the explanations of the NIPS scale parameters affected the nurses' ability to assess pain in newborns (Figure 1).

In evaluation 1 , the nurses' ability to observe the presence of the pain was 


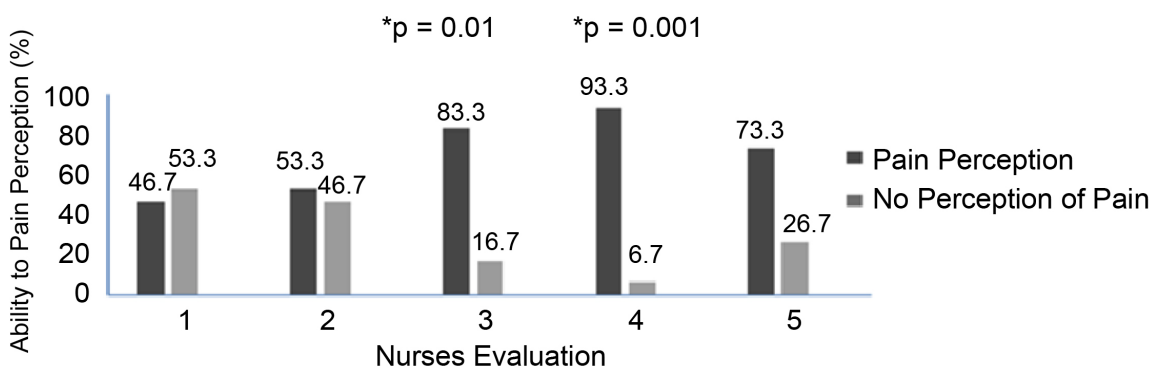

Figure 1. Variation in the frequency of correctly and incorrectly perception of neonatal pain in evaluations $1,2,3,4$ and 5 .

$46.7 \%(\mathrm{n}=14)$; in evaluation 2 , the rate was $53.3 \%(\mathrm{n}=16)$. There was no significant difference between the results of evaluation 1 and 2 (McNemar Test, $\mathrm{p}=$ 0.77).

Comparing the ratings 1 and 3 , before and after being provided clarification on the NIPS, $46.7 \%$ of nurses $(n=14)$ were able to detect the presence of pain, there was an increase this percentage to $83.3 \%(n=25)$. There was a statistically significant difference to this perception (McNemar test, $\mathrm{p}=0.01$ ). Likewise, when comparing the ratings 1 and 4, there is an increase in the ability of nurses to identify the presence of pain of $46.7 \%(n=14)$ to $93.3 \%(n=28)$, noting difference was statistically significant in increasing the ability to observe the presence of the symptom (McNemar test, $\mathrm{p}=0.001)$.

\section{Discussion}

Thirty nurses (average age $32.9 \pm 6.9$ years) took part in this investigation. Authors report that the accuracy of health professionals' evaluation of pain in children increases with the age of the observer [11]. The influence of age of the observer was not found when the pain of pediatric patients is evaluated [12].

None of the professionals in this study had a specialized background in either pediatrics or neonatology. The professional experience of the nurses in this study reported among seven and nine years. Professional assessment of pain in newborns can be influenced by personal, professional, and socioeconomic characteristics. The professional experience and personal and socio-economic characteristics of the professional responsible for neonatal care may influence their perception of pain [13]. Health professionals who have received more instruction are better able to precisely evaluate pain [13] [14]. Health professionals and caregivers with more experience are more sensitive to the presence and intensity of pain [14]. However similarly to this research Breau et al. verified the length of professional experience does not influence the observer's interpretation or recognition of pain [15].

We verified that the majority of the nurses in this study did not know of any scale that could be used to evaluate pain in newborns. Very few of the nurses knew thereof more than one scale. These findings were comparable to those reported elsewhere. Others authors reported on the difficulty of health profession- 
als to evaluate pain over this age range, while such evaluation is essential for instituting appropriate treatment [1] [16].

The neonatal pain should be routinely monitored, evaluated, reviewed and documented clearly to facilitate the treatment and promote its relief and the use of pain scale is one of the strategies recommended for this intervention [9] [16].

This study showed that nurses' ability to evaluate pain was improved by an explanation of the parameters of the NIPS scale. When the results of evaluations 1 and 2 (before the explanation of the NIPS scale parameters) were compared to those of evaluations 3, 4 and 5 (after explanation), we observed an increase in the frequency of the correct diagnosis of the presence of pain. As it was demonstrated previously signs of pain, but may be those most in need for appropriate intervention [17].

At-work education programs can successfully improve workers' practical skills and help create more capable health professionals. In the nursing profession, the process of education is fundamental to producing qualified professionals and ensuring that the highest possible level of care is achieved [18]. The author suggest that such a process favors personal development and raises the level of professional satisfaction as productivity improves.

\section{Conclusions}

This study showed that even though the occurrence of pain in the neonatal period was recognized, professionals had no knowledge of scales available for its evaluation.

The study described a clear increase in the number of professionals who were able to identify the presence of pain after they had received explanations of the parameters of the NIPS scale of infant pain evaluation. This result affirms the importance of team training techniques and suggests that this tool should be used consistently so that pain can be evaluated and treated systematically.

It is absolutely necessary to invest in the preparation of these professionals to improve the technical capacity of the unit as well as individual nurses' sensibility and understanding of how to treat neonatal pain.

\section{References}

[1] Lalitha, K. (2013) Pain Relief in Neonates. Journal of Neonatal Surgery, 2, 19.

[2] Holsti, L., Grunau, R.E. and Shany, E. (2011) Assessing Pain in Preterm Infants in the Neonatal Intensive Care Unit: Moving to a "Brain-Oriented" Approach. Pain Management, 1, 171-179. https://doi.org/10.2217/pmt.10.19

[3] Johnston, C., Campbell-Yeo, M., Fernandes, A., Inglis, D., Streiner, D. and Zee, R. (2014) Skin-to-Skin Care for Procedural Pain in Neonates. Cochrane Database Syst Rev., 1. https://doi.org/10.1002/14651858.CD008435.pub2

[4] Polomano, R.C., Dunwoody, C.J., Krenzischek, D.A., et al. (2008) Perspective on Pain. Management in the 21st Century. Pain Management Nursing, 9, S3-S10. https://doi.org/10.1016/j.pmn.2007.11.002

[5] Ulrich-Lai, Y.M. and Herman, J.P. (2009) Neural Regulation of Endocrine and Au- 
tonomic Stress Responses. Nature Reviews Neuroscience, 10, 397-409. https://doi.org/10.1038/nrn2647

[6] Hall, R.W. and Anand, K.J. (2014) Pain Management in Newborns. Clinics in Perinatology, 41, 895-924. https://doi.org/10.1016/j.clp.2014.08.010

[7] American Academy of Pediatrics Committee on Fetus and Newborn, Section on Surgery, and Section on Anesthesiology and Pain Medicine; Canadian Paediatric Society Fetus and Newborn Committee. Prevention and Management of Pain in the Neonate: An Update. Pediatrics, 118, 2231-2241.

[8] Stevens, B.J., Gibbins, S., Yamada, J., Dionne, K., Lee, G., Johnston, C. and Taddio, A. (2014) The Premature Infant Pain Profile-Revised (PIPP-R): Initial Validation and Feasibility. The Clinical Journal of Pain, 30, 238-243. https://doi.org/10.1097/AJP.0b013e3182906aed

[9] Witt, N., Coynor, S., Edwards, C. and Bradshaw, H. (2016) A Guide to Pain Assessment and Management in the Neonate. Current Emergency and Hospital Medicine Reports, 4, 1-10. https://doi.org/10.1007/s40138-016-0089-y

[10] Hudson-Barr, D., Capper-Michel, B., Lambert, S., Palermo, T.M., Morbeto, K. and Lombardo, S. (2002) Validation of the Pain Assessment in Neonates (PAIN) Scale with the Neonatal Infant Pain Scale (NIPS). Neonatal Network, 21, 15-21.

https://doi.org/10.1891/0730-0832.21.6.15

[11] Torres, C.A., Bartley, E.J., Wandner, L.D., Alqudah, A.F., Hirsh, A.T. and Robinson, M.E. (2013) The Influence of Sex, Race, and Age on Pain Assessment and Treatment Decisions Using Virtual Human Technology: A Cross-National Comparison. Journal of Pain Research, 6, 577-588.

[12] Balda, R.C.X., Guinsburg, R., Almeida, M.F.B., et al. (2000) The Recognition of Facial Expression of Pain in Full-Term Newborns by Parents and Health Professionals. Arch Pediatr Adolesc Med, 154, 1009-10016. https://doi.org/10.1001/archpedi.154.10.1009

[13] Finley, G.A., Kristjánsdóttir, O. and Forgeron, P.A. (2009) Cultural Influences on the Assessment of Children's Pain. Pain Research and Management, 14, 33-37. https://doi.org/10.1155/2009/763031

[14] Pillai Riddell, R. and Racine, N. (2009) Assessing Pain in Infancy: The Caregiver Context. Pain Research and Management, 14, 27-32. https://doi.org/10.1155/2009/410725

[15] Breau, L.M., Mcgrat, H.P.J., Stevens, B., et al. (2006) Judgments of Pain in the Neonatal Intensive Care Setting: A Survey of Direct Care Staffs' Perceptions of Pain in Infants at Risk for Neurological Impairment. The Clinical Journal of Pain, 22, 122129. https://doi.org/10.1097/01.ajp.0000154045.45402.ec

[16] Walter-Nicolet, E., Annequin, D., Biran, V., Mitanchez, D. and Tourniaire, B. (2010) Pain Management in Newborns: From Prevention to Treatment. Pediatric Drugs, 12, 353-365. https://doi.org/10.2165/11318900-000000000-00000

[17] Anand, K.J., Bergqvist, L.L., Hall, R.W. and Carbajal, R. (2011) Acute Pain Management in Newborn Infants. Pain: Clinical Updates, XIX, 1-6.

[18] Badr, L.K., Abdallah, B., Hawar, M., Sidani, S., Kassar, M., Nakad, P., et al. (2010) Determinants of Premature Infant Pain. Responses to Heel Sticks. Pediatric Nursing, 36, 129-136. 